Journal of Bangladesh Academy of Sciences, Vol. 36, No. 2, 147-152, 2012

\title{
ON GLOBAL EXISTENCE THEOREM OF CERTAIN VOLTERRA INTEGRAL EQUATION OF SECOND KIND
}

\author{
MD. SHARIFUL ISLAM, MIR SHARIFUL ISLAM ${ }^{* 1}$ AND \\ A.F.M. KHODADAD KHAN
}

Independent University, Dhaka-1212, Bangladesh

\begin{abstract}
The aim of the paper was to fabricate an alternative proof of a global existence theorem of certain type of Volterrea integral equation on the basis of the hypothesis. The new proof has been given by constructing suitable function space and using fixed point theorem. Relaxing some hypotheses in the same and using Bielecki's notion of norm another global existence theorem has been proposed and proved.
\end{abstract}

Key words: Global Existence Theorem, Certain Volterra Integral Equation

\section{INTRODUCTION}

Consider the Volterra integral equation of the second kind in $R^{n}$

$$
x(t)=f(t)+\int_{0}^{t} k(t, s) g(s, x(s)) d s
$$

where the given function $f(t)$ and the kernel $k(t, s)$ are assumed to be continuous on the interval $[0, \infty)$ and the extended triangular region $0 \leq s<t<\infty$, respectively. Moreover there exists $\quad M>0$ such that $\int_{0}^{t}|k(t, s)| d s \leq M, t \geq 0$ and $g(t, x)$ is continuous on $[0, \infty) \times R^{n},|g(t, x)-g(t, y)| \leq L|x-y|, t \geq 0$ where $M L<1$ is bounded on $[0, \infty)$. Where |. means supremum norm of vector and matrix.

By employing the constructing uniformly convergent sequence of functions (successive approximation) as in Corduneanu (1969), it can be shown (1) possesses a unique global continuous solution $x(t)$ of equation (1) on the interval $[0, \infty)$. On the other hand the present authors applied Banach contraction mapping principle to (1), by formulating suitable function space under the same set of hypotheses. The operator $T \phi(t)=f(t)+\int_{0}^{t} k(t, s) g(s, \phi(s)) d s ; t \geq 0$ takes $B$ into $B$, where $B$ is the space of all real valued continuous function on $[0, \infty)$. The operator $T$ is not, in general, contracting unless the product $M L<1$. Hence to apply the contraction principle to $T$, it is necessary to restrict either the highest possible mass of the kernel or the Lipschitz's constant in the second argument of $g(t, x) . L$ and $M$ may be interpreted otherwise. In second case, if the authors relax the hypotheses $M L<1$ and define the norm of a function using Bielecki

* Corresponding author:<mirsharifuldu77@yahoo.com>.

${ }^{1}$ University of Information Technology and Sciences, Dhaka-1212. 
Idea in (Miller 1973), we can show, at the influence of Miller (1973), that (1) has unique continuous solution on $[0, \infty)$ (Strauss 1970).

Global existence theorem (1): Corduneanu (1969) described a global existence theorem under the following hypotheses. The present authors proved the same using contraction mapping theorem instead constructing sequence of functions.

Consider the Volterra integral equation $x(t)=f(t)+\int_{0}^{t} k(t, s) g(s, x(s)) d s$

Assume that (1) satisfies the following conditions:

(i) $f(t)$ is continuous and bounded on $[0, \infty)$.

(j) $k(t, s)$ is continuous on $0 \leq s<t<\infty$ and there exists $M>0$ such that $\int_{0}^{t}|k(t, s)| d s \leq M, t \geq 0$.

(k) $g(t, x)$ is continuous on $[0, \infty) \times R^{n}$ and $|g(t, x)-g(t, y)| \leq L|x-y|, t \geq 0$ where $L<M^{-1}$ or $M<L^{-1} \Rightarrow M L<1$.

(1) $g(t, 0)$ is bounded on $[0, \infty)$, i.e. $|g(t, 0)|<m_{2}<\infty ; t \in[0, \infty)$

then there exists a unique continuous bounded solution $x(t)$ of $(1)$ on $[0, \infty)$.

Proof: Let $B$ be the Banach space of bounded continuous functions on $[0, \infty)$ with supremum norm |. (Simmons 2004), for $\phi \in B,|\phi|=\underset{0 \leq t<\infty}{\operatorname{Sup}}|\phi(t)|$.

For each $\phi \in B$, we define the mapping $\phi \mapsto T \phi$ by

$T \phi(t)=f(t)+\int_{0}^{t} k(t, s) g(s, \phi(s)) d s ; t \geq 0$.

Now, $|T \phi(t)| \leq|f(t)|+\int_{0}^{t}|k(t, s) \| g(s, \phi(s))| d s ; t \geq 0$.

Given, $|f(t)| \leq m_{1}<\infty$

$|T \phi(t)| \leq m_{1}+\int_{0}^{t}\left|k(t, s)\left\|g(s, \phi(s))\left|d s-\int_{0}^{t}\right| k(t, s)\right\| g(s, 0)\right| d s+\int_{0}^{t}|k(t, s) \| g(s, 0)| d s$

$\leq m_{1}+\int_{0}^{t}|k(t, s)||g(s, \phi(s))-g(s, 0)| d s+\int_{0}^{t}|k(t, s) \| g(s, 0)| d s$

But $|g(t, 0)|<m_{2}<\infty ; t \in[0, \infty)$ and $|g(s, \phi(s))-g(s, 0)|<L|\phi(s)-0|$

$|T \phi(t)| \leq m_{1}+L \int_{0}^{t}|k(t, s) \| \phi(s)| d s+m_{2} \int_{0}^{t}|k(t, s)| d s$

$\leq m_{1}+|\phi| L \int_{0}^{t}|k(t, s)| d s+m_{2} \int_{0}^{t}|k(t, s)| d s$ and $\int_{0}^{t}|k(t, s)| d s<M<\infty$

$\leq m_{1}+|\phi| L M+m_{2} M<\infty$

Again $|T \phi(t+h)-T \phi(t)| \rightarrow 0$ as $h \rightarrow 0$ 


$$
\begin{aligned}
& \quad|T \phi(t+h)-T \phi(t)|= \\
& \left|f(t+h)-f(t)+\int_{0}^{t+h} k(t+h, s) g(s, \phi(s)) d s-\int_{0}^{t} k(t, s) g(s, \phi(s)) d s\right| \\
& \quad \leq|f(t)-f(t+h)|+\underset{0 \leq s \leq t+h}{\operatorname{Max}}|g(s, \phi(s))|\left|\int_{0}^{t+h} k(t+h, s) d s-\int_{0}^{t} k(t, s) d s\right| \\
& \quad \leq|f(t)-f(t+h)|+\underset{0 \leq s \leq t+h}{\operatorname{Max}}|g(s, \phi(s))|\left|\int_{0}^{t}(k(t+h, s)-k(t, s)) d s+\int_{t}^{t+h} k(t+h, s) d s\right| \\
& \left.\quad \leq|f(t)-f(t+h)|+\underset{0 \leq s \leq t+h}{\operatorname{Max}}|g(s, \phi(s))|\left|\int_{0}^{t}\right|(k(t+h, s)-k(t, s))\left|d s+\int_{t}^{t+h}\right| k(t+h, s) \mid d s\right] \\
& \quad f(t) \text { and } k(t, s) \text { are continuous on } t \in[0, \infty) \\
& \quad \leq 0, \text { as } h \rightarrow 0 .
\end{aligned}
$$

Therefore, $T \phi$ is bounded and continuous. Hence, $T \phi: B \rightarrow B$.

For $\phi$ and $\psi \in B$

$$
\begin{aligned}
& |T \phi(t)-T \psi(t)| \leq\left|\int_{0}^{t} k(t, s) g(s, \phi(s)) d s-\int_{0}^{t} k(t, s) g(s, \psi(s)) d s\right| \\
& |T \phi(t)-T \psi(t)| \leq \int_{0}^{t}|k(t, s) \| g(s, \phi(s))-g(s, \psi(s))| d s \\
& |T \phi(t)-T \psi(t)| \leq L \int_{0}^{t}|k(t, s)||\phi(s)-\psi(s)| d s \\
& |T \phi-T \psi| \leq L|\phi-\psi| \int_{0}^{t}|k(t, s)| d s \\
& |T \phi-T \psi| \leq M L|\phi-\psi| \text { But } M L<1
\end{aligned}
$$

$T: B \mapsto B$ is a contraction mapping. By Banach contraction mapping theorem $T$ has unique fixed point in $B$. Therefore (1) has a global continuous bounded solution under considered settings.

Global existence theorem (2):Here, relaxing the hypothesis (k) in the previous theorem and defining the norm of the function using Bielecki ideas in Sherwood et al. (1964], the authors have established the following global existence theorem:

Consider the Volterra integral equation $x(t)=f(t)+\int_{0}^{t} k(t, s) g(s, x(s)) d s$

Assume that (1) satisfies the following conditions

(i) $f(t)$ is continuous and bounded on $[0, \infty)$.

(j) $k(t, s)$ is continuous on $0 \leq s<t<\infty$ and there exists $M>0$ such that $\int_{0}^{t}|k(t, s)| d s \leq M, t \geq 0$. 
(k) $g(t, x)$ is continuous on $[0, \infty) \times R^{n}$ and $|g(t, x)-g(t, y)| \leq L|x-y|, t \geq 0$.

(1) $g(t, 0)$ is bounded on $[0, \infty)$

then there exists a unique continuous bounded solution $x(t)$ of $(1)$ on $[0, \infty)$.

Proof: Let $B_{\lambda}$ be the Banach space of the bounded continuous function $[0, \infty)$ with the norm $|$.$| , where for \phi \in B_{\lambda},|\phi|=\underset{0 \leq t<\infty}{\operatorname{Sup}}|\phi(t)| e^{-\lambda t}<\infty, \lambda>0$ for each $\phi \in B_{\lambda}$ (Corduneanu 1973).

We define the mapping $\phi: \mapsto T \phi$ by

$$
\begin{aligned}
& T \phi(t)=f(t)+\int_{0}^{t} k(t, s) g(s, \phi(s)) d s ; t \geq 0 \\
& \begin{aligned}
|T \phi(t)| & \leq|f(t)|+\int_{0}^{t}|k(t, s) \| g(s, \phi(s))| d s ; t \geq 0 \\
& =|f(t)|+\int_{0}^{t}\left|k(t, s)\left\|g(s, \phi(s))-g(s, 0)\left|d s+\int_{0}^{t}\right| k(t, s)\right\| g(s, 0)\right| d s ; t \geq 0
\end{aligned} \\
& T \phi(t) \leq|f(t)|+L \int_{0}^{t}|k(t, s)||\phi(s)-0| d s+\int_{0}^{t}|k(t, s) \| g(s, 0)| d s ; t \geq 0 \\
& T \phi(t) \leq|f(t)|+L \int_{0}^{t}|k(t, s)| \frac{|\phi(s)|}{e^{\lambda s}} e^{\lambda s} d s+\int_{0}^{t}|k(t, s) \| g(s, 0)| d s ; t \geq 0 \\
& T \phi(t) \leq|f(t)|+L|\phi| e^{\lambda t} \int_{0}^{t}|k(t, s)| d s+\int_{0}^{t}|k(t, s) \| g(s, 0)| d s ; t \geq 0 \\
& T \phi(t) e^{-\lambda t} \leq|f(t)| e^{-\lambda t}+L|\phi| \int_{0}^{t}|k(t, s)| d s+e^{-\lambda t} \int_{0}^{t}|k(t, s) \| g(s, 0)| d s ; t \geq 0
\end{aligned}
$$

Taking supremum on both sides for $0<t<\infty$

$$
|T \phi| \leq m_{1}+L M|\phi|+m_{2} M \text {, where }|f(t)| \leq m_{1} \text { and }|g(t, 0)| \leq m_{2}
$$

Therefore $T \phi(t)$ is bounded on $0 \leq t<\infty$ and

$$
\begin{aligned}
& |T \phi(t+h)-T \phi(t)|= \\
& \left|f(t+h)-f(t)+\int_{0}^{t+h} k(t+h, s) g(s, \phi(s)) d s-\int_{0}^{t} k(t, s) g(s, \phi(s)) d s\right| \\
& |T \phi(t+h)-T \phi(t)|= \\
& \leq|f(t+h)-f(t)|+\int_{0}^{t}\left|k(t+h, s)-k(t, s)\left\|g(s, \phi(s))\left|d s+\int_{t}^{t+h}\right| k(t+h, s)\right\| g(s, \phi(s))\right| d s
\end{aligned}
$$

But, $\operatorname{Max}\left\{|g(s, \phi(s))| \leq m_{3}, 0 \leq s \leq t\right\} ; m_{3}>0$.

$$
\operatorname{Max}\left\{|g(s, \phi(s))| \leq m_{4}, t \leq s \leq t+h\right\} ; m_{4}>0
$$




$$
\begin{aligned}
& \operatorname{Max}\left\{|k(t+h, s)| \leq m_{5}, t \leq s \leq t+h\right\} ; m_{5}>0 . \\
& |f(t+h)-f(t)| \rightarrow 0 \text { as } h \rightarrow 0 \text { and } \\
& |k(t+h, s)-k(t, s)| \rightarrow 0 \text { as } h \rightarrow 0 \text { therefore } \\
& |T \phi(t+h)-T \phi(t)| \leq m_{4} m_{5} h \\
\Rightarrow & e^{-\lambda t}|T \phi(t+h)-T \phi(t)| \leq m_{4} m_{5} h \\
\Rightarrow & e^{-\lambda t}|T \phi(t+h)-T \phi(t)| \rightarrow 0 \text { as } h \rightarrow 0 .
\end{aligned}
$$

Therefore $T \phi$ is continuous and $T \phi \in B_{\lambda}$ which implies $T: B_{\lambda} \mapsto B_{\lambda}$

For any $\phi, \psi \in B_{\lambda}$

$$
\begin{aligned}
& |T \phi(t)-T \psi(t)| \leq L \int_{0}^{t}|k(t, s)||\phi(s)-\psi(s)| d s \\
& \Rightarrow|T \phi(t)-T \psi(t)| \leq L \int_{0}^{t}|k(t, s)| e^{-\lambda s}|\phi(s)-\psi(s)| e^{\lambda s} d s \\
& \Rightarrow|T \phi(t)-T \psi(t)| \leq L M|\phi-\psi| \int_{0}^{t} e^{\lambda s} d s \\
& \Rightarrow|T \phi(t)-T \psi(t)| \leq L M|\phi-\psi| \frac{1}{\lambda}\left(e^{\lambda t}-1\right) \\
& \Rightarrow|T \phi(t)-T \psi(t)| \leq L M|\phi-\psi| \frac{1}{\lambda} e^{\lambda t} \\
& \Rightarrow|T \phi-T \psi| \leq L M|\phi-\psi| \frac{1}{\lambda}
\end{aligned}
$$

Here, we choose $\lambda$ large enough so that $L M / \lambda<1 \Rightarrow L M<\lambda$. Therefore, for $L M<\lambda$,

$T: B_{\lambda} \mapsto B_{\lambda}$ is a contraction mapping. By Banach contraction mapping theorem $T$ has unique fixed point in $B_{\lambda}$. Therefore (1) has a global continuous bounded solution under considered settings.

\section{CONCLUSION}

If $\lambda$ is taken zero both the function spaces in the theorems (1) and (2) becomes identical and second theorem is no longer valid without the hypothesis (k) and $L M<1$ as in the first theorem. 


\section{REFERENCES}

Corduneanu, C. 1969. Principles of Differential and Integral Equations. Boston: Allyn and Beacon.

Corduneanu, C. 1973. Integral Educations and Stability of Feedback Systems. Mathematics in Science and Engineering. 104. Academic press.New York and London.

Miller, R.K. 1971. Nonlinear Volterra Integral Equations. New York: W. A. Benjamin, inc.

Sherwood, N. D., C. Chu and J. B. Diaz. 1964. A fixed point theorem for (in the large) application of the contraction principle.Presentata dal socio corrisp. Gaetano fichlranell' adunanza.

Simmons,G.F. 2004. Topology and Modern Analysis. Addison-Wesley. Pearson education. McGraw-Hill. Kogakusha Ltd.

Strauss, A. 1970. On a perturbed volterra integral equation. Journal of mathematical analysis and applications 30: 564-575.

(Received revised manuscript on 16 April, 2012) 